\title{
Trace Element Analysis under 100 ppm and Chemical State Analysis in Small Area using Wavelength Dispersive Soft X-ray Emission Spectrometer in FE-SEM
}

\author{
H. Takahashi ${ }^{1}$, S. Asahina ${ }^{2}, \quad$ M. Terauchi ${ }^{3}$, C. Nielsen ${ }^{4}$, P. McSwiggen ${ }^{4}$ \\ ${ }^{1 .}$ Global business promotion division, JEOLLtd., 13F, Otemachi Nomura Bld. 2-1-1 Otemachi, Chiyoda-ku, Tokyo, \\ 100-0004 Japan \\ 2. SM business unit JEOLLtd., 1-2 Musashino, 3-chome, Akishima, Tokyo 196-8558, Japan. \\ 3. Institute for Multidisciplinary Research for Advanced Materials, Tohoku University, Sendai 980-8577, Japan. \\ ${ }^{4}$.JEOL USA, 11 Deabom Rd. Peabody, MA 01960, ISA.
}

A novel wavelength dispersive soft X-ray emission spectrometer (WD-SXES) has been developed. It covers nominally the X-ray energy range between 50 and $210 \mathrm{eV}[1,2]$ using two kinds of gratings. One of the characteristic features of the WD-SXES is its parallel detection of the signal. This allows it to be used like a conventional energy dispersive spectrometer. A second feature is its high energy resolution, which is about $0.2 \mathrm{eV}$ for Al-L emission. This resolution is comparable to that of X-ray photoelectron spectroscopy or electron energy-loss spectroscopy. This enables us the ability to obtain important information about chemical bonding in bulk samples from observed spectra as a result of the very high- energy resolution. We have already reported a few examples obtained with the WD-SXES [3]. One important feature of the WD-SXES is that it can detect the Li-K emission spectrum. In the case of an anode electrode from a lithium ion battery (LIB), two types of lithium peaks are observed; one lower energy peak at $50 \mathrm{eV}$, from the valence band, and the other higher energy peak at $54 \mathrm{eV}$, from the core loss. In addition, we have documented the ability to measure various kinds of X-ray spectra of K, L, M and $\mathrm{N}$ emission from Lithium to Uranium [4].

In the modern FE-SEM, a wide variety of attachments are used, including multi-SDD, EBSD, WDS, many kinds of electron signal detectors, sputtering tools, low vacuum devices, cathodeluminescence detectors, etc. These are very important for characterizing a wide range of materials. Now, due to its good peak to background ratio and high sensitive CCD camera, the WD-SXES would be an ideal attachment for trace element analysis. Combining the WD-SXES with a FE-SEM, the higher spatial resolution and high sensitivity can provide new insights in material science. For example, the SEM is very useful for observing the distribution of microstructures for metals and ceramics. We have also demonstrated that trace carbon contents can be detected in concentrations from $67 \mathrm{ppm}$ to $8700 \mathrm{ppm}$ using low alloy steel NBS (NIST) series from 1261a to 1265a respectively. Backscattered electron images of these materials, along with their trace carbon peaks are shown in Figure 1 (a) and (b). In addition, from the peak shape of the $\mathrm{C}-\mathrm{K} \alpha$ spectra, it is possible to distinguish a carbide grain from graphite, as shown in Figure 2. Using a FE-SEM, we can observe tiny grains or grain boundaries, and by combining it with the WD-SXES, we can analyze these features for trace carbon abundances. It is also possible to analyze for trace boron and nitrogen. In this presentation, we will discuss the possibilities for using the WD-SXES for trace elemental analyses and chemical state analyses.

\section{References:}

[1] M. Terauchi, et al., J. Electron Microscopy, 61, 1 (2012).

[2] T. Imazono, et al., Appl. Opt. 51, 2351 (2012).

[3] H. Takahashi, et al., Microscopy and Microanalysis, 19 (supple. 2), 1258 (2013).

[4] H. Takahashi, et al., Microscopy and Microanalysis, 20 (supple. 3), 684 (2014) 
(a)
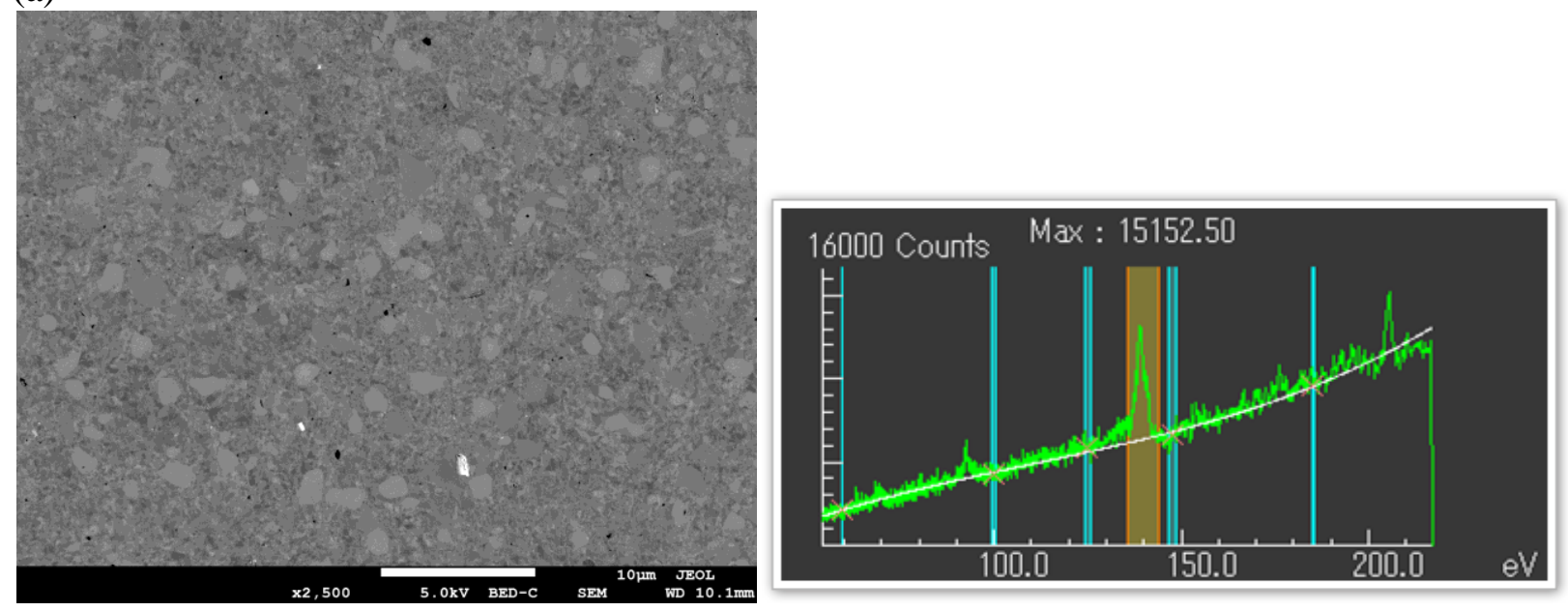

(b)
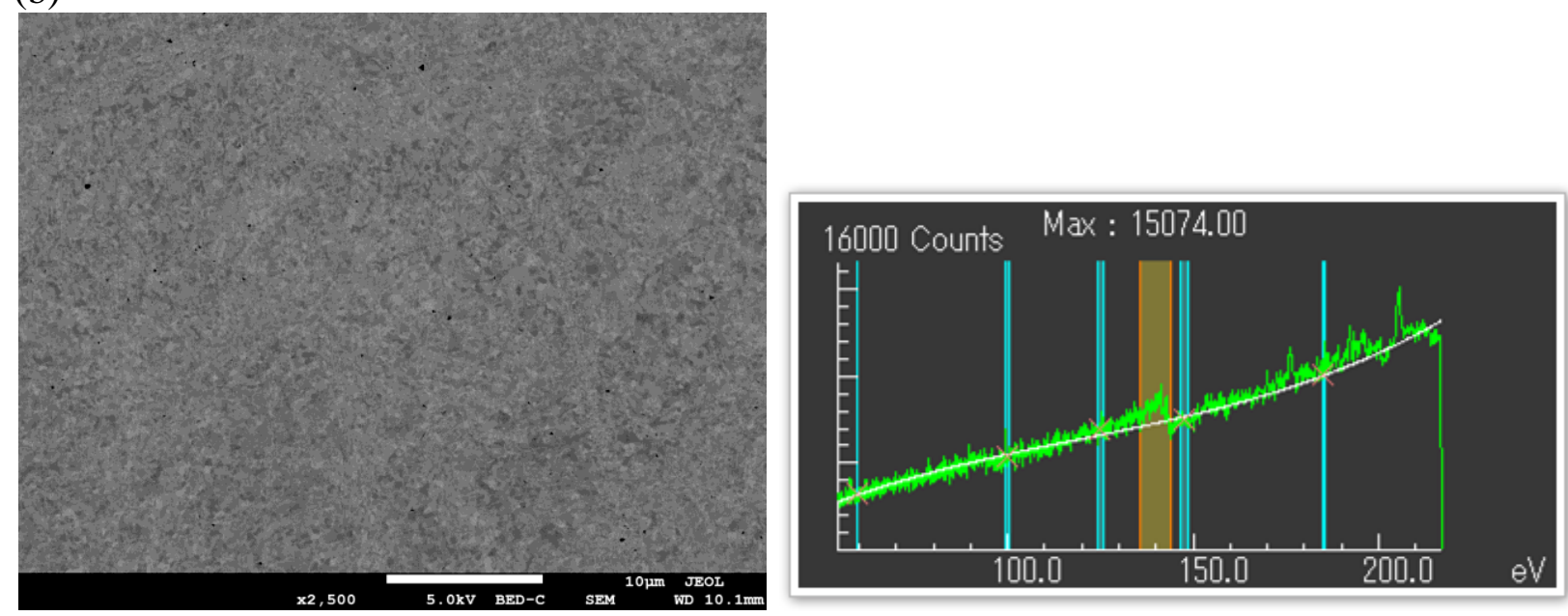

Figure 1. Backscattered electron images and C-K $\alpha$ peaks using JSM-7800FPrime+SXES for (a) 8700 ppm and (b) 67 ppm carbon in low alloy steel NBS (NIST) 1264a and 1265a

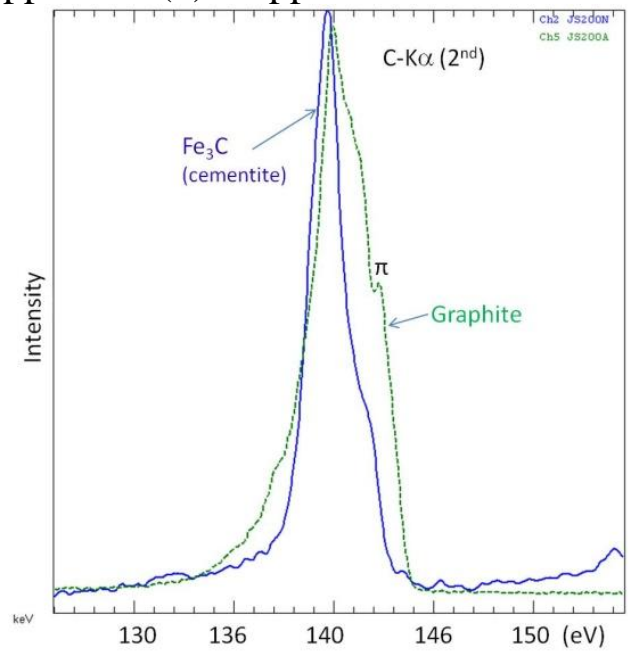

Figure 2. Comparison of $\mathrm{C}-\mathrm{K} \alpha$ spectra between $\mathrm{Fe}_{3} \mathrm{C}$ (Solid blue line) and graphite (Dash green line) 